\title{
Care of the Child With Ebola Virus Disease*
}

\author{
Carl O. Eriksson, MD, MPH${ }^{1}$; Timothy M. Uyeki, MD, MPH, MPP²; Michael D. Christian, MD, MSc ${ }^{3}$; \\ Mary A. King, MD, $\mathrm{MPH}^{4}$; Dana A. V. Braner, $\mathrm{MD}^{1}$; Robert K. Kanter, MD ${ }^{5,6}$; Niranjan Kissoon, $\mathrm{MD}^{7}$
}

Objectives: To provide clinicians with practical considerations for care of children with Ebola virus disease in resource-rich settings. Data Sources: Review of the published medical literature, World Health Organization and government documents, and expert opinion. Data Synthesis: There are limited data regarding Ebola virus disease in children; however, reported case-fatality proportions in children are high. Ebola virus may affect immune regulation and endothelial function differently in children than adults. Considerations for care of children with Ebola virus disease are presented. Conclusions: Ebola virus disease is a severe multisystem disease with high mortality in children and adults. Hospitals and clinicians must prepare to provide care for patients with Ebola virus disease before such patients present for care, with particular attention to rigorous infection control to limit secondary cases. Although there is no proven specific treatment for Ebola virus disease, meticulous supportive care offers patients the best chance of survival. (Pediatr Crit Care Med 2015; 16:97-103)

\section{*See also p. 184.}

'Division of Pediatric Critical Care, Department of Pediatrics, Oregon Health \& Science University, Portland, OR.

2Influenza Division, National Center for Immunization and Respiratory Diseases, U.S. Centers for Disease Control and Prevention, Atlanta, GA.

${ }^{3}$ Department of Critical Care, Mount Sinai Hospital, Toronto, ON, Canada.

${ }^{4}$ Pediatric Critical Care Medicine, Seattle Children's Hospital and Harborview Medical Center, Seattle, WA.

${ }^{5}$ Pediatric Critical Care Medicine, Virginia Tech Carilion School of Medicine, Roanoke, VA.

${ }^{6}$ National Center for Disaster Preparedness, Columbia University, New York, NY. 'Departments of Pediatrics and Emergency Medicine, British Columbia's Children's Hospital, Vancouver, BC, Canada.

This work was performed at Oregon Health \& Science University, Portland, OR. Dr. Uyeki disclosed government work. Dr. Christian received support for article research and disclosed government work. Dr. Kanter disclosed past employment with SUNY Updated Medical University and current employment with the Virginia Tech Carilion School of Medicine. He received support for travel from the National Center for Disaster Preparedness, Columbia University; the American College of Chest Physicians; and the NYS DOH Task Force on Life and the Law. His institution consulted for the National Center for Disaster Preparedness, Columbia University; and received grant support from the Baton Rouge Area Foundation. The remaining authors have disclosed that they do not have any potential conflicts of interest.

This content is solely the responsibility of the authors and does not necessarily represent the official views of the Centers for Disease Control and Prevention.

For information regarding this article, E-mail: eriksson@ohsu.edu

Copyright (C) 2015 by the Society of Critical Care Medicine and the World Federation of Pediatric Intensive and Critical Care Societies

DOI: 10.1097/PCC.0000000000000358
Key Words: adolescent; child; critical care; Ebola hemorrhagic fever; Ebola virus; Ebola virus disease; infant; sepsis

1 bola virus infection causes a severe disease characterized by multisystem involvement and high mortality. Since the first recognized epidemics of Ebola virus in 1976, the virus has been responsible for sporadic outbreaks in sub-Saharan Africa. In March 2014, the World Health Organization was notified of an outbreak of Ebola virus disease (EVD) in southwestern Guinea, subsequently attributed to Zaire ebolavirus (EBOV). This ongoing outbreak likely began in Guinea in December 2013 (1) and constitutes the largest Ebola virus epidemic to date with further spread to Liberia, Sierra Leone, Nigeria, Senegal, Mali, Spain, the United Kingdom, and the United States through December 2014.

Most patients with EVD have been treated in very resourcepoor settings, with interventions limited to basic supportive care; therefore, it is difficult to estimate the potential benefit of modern critical care. To date, a small number of adult patients have been treated in the United States and several European countries. Most published clinical data are derived from adult patients. There is limited knowledge about EVD in children; however, reported case-fatality proportions in children are high $(2,3)$.

The objective of this report is to provide clinicians with practical considerations for care of children with EVD in resource-rich settings.

\section{TRANSMISSION OF EBOLA VIRUS}

The natural reservoir for Ebola viruses is believed to be fruit bats, with infected mammals such as duiker antelopes and nonhuman primates having high mortality (4-6). Ebola virus particles or viral RNA have been detected in skin and body fluids of humans or nonhuman primates. Ebola viruses can enter the body through mucosal surfaces, skin breaks, or parenteral introduction and appear to initially infect monocytes, macrophages, and dendritic cells, which then disseminate the infection (7). Direct contact with infected animals can result in zoonotic transmission with subsequent spread among humans who had direct contact with blood or bodily fluids of infected persons. To date, five species of Ebola virus have been identified (Zaire, Sudan, Bundibugyo, Reston, and Taï Forest) with variable virulence; case-fatality proportions during outbreaks for EBOV infection range from $59 \%$ to $88 \%(8,9)$, whereas there 
are no known fatalities from Taï Forest ebolavirus infection, and Reston ebolavirus is not known to cause human disease.

\section{PATHOPHYSIOLOGY OF EVD}

Ebola virus has tropism for multiple tissues, including the liver and adrenal gland, causing hepatocellular and adrenocortical necrosis (7). In addition, infection triggers a dysregulated immune response that includes expression of several inflammatory mediators and inhibition of type I interferon response (10-12). Recovery from ebolavirus infection is associated with a well-regulated inflammatory response characterized by early Ebola-specific IgM and IgG production; in contrast, fatal cases are associated with an impaired response characterized by increased expression of several proinflammatory cytokines, lymphocyte apoptosis, marked CD8(+) lymphopenia, and lack of neutralizing antibody production (13-16). Fatal cases are also associated with high levels of ferritin, nitric oxide, and thrombomodulin, and higher levels of viremia are associated with elevated tissue factor and tissue plasminogen activator $(13,16,17)$. Immune dysregulation has led to concern about bacterial superinfection (18).

Ebola virus may affect immune regulation and endothelial function differently in children than in adults, and markers associated with survival differ between children and adults. Children infected with Sudan ebolavirus have greater elevation of interleukin (IL)-10 and the chemokine regulated on activation, normal T-cell expressed and secreted (RANTES) marker. Fatal Sudan ebolavirus cases in childhood were associated with higher IL-10 and lower RANTES levels than children who survived, while levels of IL-10 and RANTES had no association with outcomes in adults (2). Children also had higher levels of several markers of endothelial overactivation, and elevations in these markers were associated with fatal cases in children but not in adults (2). This combination of immune dysregulation and endothelial damage shares several features with severe sepsis (19).

\section{CLINICAL FEATURES OF EVD}

Although EVD is classically divided into early, late, and terminal or recovery phases, considerable overlap between these phases may occur. After an incubation period of 2-21 days (mean, $11 \mathrm{~d}$ ), an acute febrile illness develops in patients; nonspecific signs and symptoms of this early phase of disease commonly include fever, headache, chills, malaise, myalgias, asthenia, vomiting, and diarrhea $(3,7,20)$.

Diarrhea can be profuse and may mimic cholera. Hiccups, conjunctivitis, and pharyngeal erythema may also develop in patients, and a nonpruritic maculopapular rash on the face and upper body may be present during early or late phases of the disease $(7,18)$. Early laboratory abnormalities are not specific to Ebola and can include leukopenia, lymphopenia, thrombocytopenia, serum aminotransferase elevation, hyperlipasemia, hyperproteinemia, elevated prothrombin and partial thromboplastin times, fibrin split products, and elevated creatinine kinase $(7,21)$.

Patients with severe disease may develop shock from hypovolemia or sepsis, multiple organ failure, and disseminated intravascular coagulation; one patient with fatal disease cared for in a tertiary care center developed significant left ventricular systolic dysfunction (21). Fewer than half of patients develop hemorrhagic manifestations, which are often in the gastrointestinal tract and may be life threatening $(3,7,21)$. Late neurologic sequelae include confusion, delirium, seizures, and coma. Patients with fatal disease typically die between day 6 and 16 of symptoms from multisystem failure, while nonfatal cases show improvement by days 6-11 (11). Viral load has been reported to be substantially higher in fatal cases compared with survivors $(17,22)$, although this difference may be less pronounced in children (2). In surviving patients, there is a prolonged convalescent period lasting up to 8 weeks (23), characterized by arthralgia, myalgia, abdominal pain, fatigue, and weakness (18, 24). Ebola virus has been cultured from the breast milk of an infected patient at illness day 15 (25). The duration of infectivity after resolution of viremia for other bodily fluids is not completely understood, but infectious virus has been recovered from survivor semen up to 82 days after symptom onset (26).

\section{CARE OF CHILDREN WITH EVD}

\section{Diagnosis of EVD}

Since early identification of potential Ebola cases is essential for implementing proper infection control measures and the early symptoms of EVD are nonspecific, clinicians should maintain a high index of suspicion for patients with both clinical findings consistent with EVD (fever and symptoms such as headache, myalgia, abdominal pain, vomiting, diarrhea, and hemorrhage) and an epidemiologic risk factor within 21 days before symptoms onset. Such risk factors include having been in a country with widespread ebolavirus transmission or having contact with a symptomatic person with $\operatorname{EVD}(27,28)$.

Suspected cases of EVD should be reported to public health officials immediately. National public health agencies can provide guidance regarding diagnosis and infection control (29, 30 ), as well as the level-4 biosafety containment laboratories required for safely diagnosing infection with high-risk organisms such as ebolavirus.

The standard method to identify acute ebolavirus infection is by real-time reverse transcription-polymerase chain reaction testing of blood specimens (22). Other methods such as antigendetection enzyme-linked immunosorbent assay (ELISA) have been used in the past. Antibodies to Ebola virus may be detected using direct IgG and IgM ELISAs or IgM capture ELISA starting 2 days after symptom onset, although patients with fatal disease may not develop detectable antibody responses (7).

Even during Ebola outbreaks, the likelihood of EVD in a febrile returned traveler is generally much lower than the likelihood of more common imported febrile illnesses. In particular, malaria is endemic in all regions where Ebola has been identified and remains the most likely diagnosis in febrile patients from these regions. Delay in the diagnosis and treatment of malaria can lead to severe or lethal disease in a short period of time. Thus, urgent malaria testing should be performed in all children suspected of having EVD (Table 1). 


\section{TABLE 1. Care of Patients With Ebola Virus Disease: Summary of Key Pediatric Considerations}

\author{
Advance planning \\ Institutions should plan and practice for the care of \\ children with suspected EVD before such patients \\ present for care \\ Diagnosis \\ Urgent malaria testing should be performed in children \\ suspected of having EVD \\ Notify local public health officials immediately regarding \\ suspected cases of EVD
}

Patient placement and infection control

If transport can be safely undertaken, patients may benefit from early transfer to high-volume pediatric centers or biocontainment units

Consider initial admission to PICU or an Ebola treatment unit led by PICU providers even for patients not yet critically ill

Meticulous attention to infection control is essential to limiting secondary cases

Only essential staff should participate in the care of patients with suspected EVD

Ebola-specific treatment

There are no data regarding the use of anti-Ebola virus treatment in children; use of experimental therapy must occur within an acceptable ethical framework and should include case reporting to facilitate assessment of effectiveness

Monitoring and supportive care

Closely monitor and replace fluid and electrolyte losses

Consider placement of central venous or peripherally inserted central catheters to minimize venipuncture

Avoid emergent and high-risk procedures (such as extracorporeal life support), which may present an unacceptable risk of healthcare worker exposure

\section{Case reporting and documentation}

Strongly consider prospective, systematic case reporting to increase knowledge of EVD in children

$\mathrm{EVD}=$ Ebola virus disease.

\section{Infection Control}

As with any emerging infectious illness, appropriate hospital infection control for Ebola virus is essential. Institutions should plan for the care of patients with emerging infectious illnesses before potentially infected patients present for care $(29,31)$; lessons and preparedness protocols from institutions that have cared for patients with EVD can provide practical guidance $(32,33)$. Hospitals should train clinical staff to identify signs and symptoms of EVD and ensure that emergency department screening includes questions regarding travel within 21 days from an affected country and possible exposure to Ebola virus for patients with symptoms compatible with EVD $(29,31,34)$. Potential Ebola cases should be isolated immediately. Risk of Ebola virus transmission to healthcare workers in resource-rich settings is expected to be much lower than in African field hospitals, where inadequate medical supplies, no running water, and no floors likely contribute to greater exposure to Ebola virus $(21,35)$. However, due to the high case fatality of EVD, lack of proven antiviral therapy, and healthcare worker infection in Spain and the United States, meticulous attention must be paid to infection control practices.

Early planning for care of patients with EVD includes taking inventory of existing resources and identifying potential areas for managing highly infectious patients. Important factors to consider include the ability to provide negative pressure isolation as aerosol-generating procedures may be necessary, the presence of a private bathroom, the ability to restrict access to the room, sufficient space to don and remove personal protective equipment safely, the ability to perform laboratory studies while minimizing healthcare provider exposure risk, and the ability to provide criti$\mathrm{cal}$ care if necessary without having to move the patient. Hospitals should consider initially admitting children with suspected EVD to the PICU even if they do not otherwise meet PICU admission criteria. Admitting patients with EVD to the more controlled and highly monitored PICU environment may allow more effective limitation of foot traffic, decrease the number of staff that must be educated in proper infection control measures, facilitate lower nurse-to-patient ratios to ensure that infection control measures are strictly followed, and minimize risky in-hospital transfers if patients deteriorate. Alternatively, hospitals may consider establishing a geographically separate Ebola treatment unit staffed by PICU nurses and physicians, as this may further limit potential exposure of other patients and healthcare workers.

Table 2 describes recommended infection control measures for patients with EVD. Guidelines from national public health agencies have changed with increased experience treating EVD in resource-rich settings and may continue to evolve. Regardless of the equipment chosen, staff must be thoroughly trained and meticulously monitored in the appropriate use of personal protective equipment, especially its removal (36). To decrease exposure risk, only essential staff should participate in the care of patients with EVD. Given the profound importance of limiting secondary cases, children with confirmed EVD may need to be separated from their caregivers. Such separation may cause significant emotional distress, which can be somewhat mitigated by frequent videoconferencing and additional psychological support.

To decrease opportunities for healthcare personnel exposure to Ebola virus, phlebotomy, procedures, laboratory testing, and the use of needles and sharps should be minimized as much as possible $(31,37,38)$. Strict adherence to recommendations for body fluid specimen collection, transport, and testing (39), as well as for environmental infection control (40), is advisable. Hospital laboratories should be notified as early as possible of the need to perform laboratory testing for a patient suspected of having EVD so that appropriate packaging and transport can be ensured. Specimens should not be sent by pneumatic tube systems. Hospitals may consider using dedicated laboratory equipment for patients with suspected EVD and keeping this equipment at the bedside 


\section{TABLE 2. Recommended Isolation Measures for Patients Suspected of Having Ebola Virus Disease, Based on U.S. and Canadian Government and World Health Organization Recommendations $(31,36-38,40,65)$}

Patient placement

Patients should be placed in single-patient rooms with private bathrooms

Consider initial placement in an airborne isolation room for any patients with suspected EVD who may need aerosol-generating procedures

Consider initial placement in PICU or geographically separate Ebola treatment unit to facilitate close monitoring, limit the pool of healthcare providers, and avoid potentially risky transfers if patients deteriorate

Facilities should establish separate spaces for donning and removing PPE; space where PPE is removed must be sufficiently large to allow ideal practices without risk of surface contamination

Nonessential contact with the patient or body fluids should be avoided

Transmission-based precautions

In addition to universal precautions, contact and droplet precautions should be used

All healthcare personnel and caregivers entering the patient room must be thoroughly trained in proper technique when donning and removing PPE; equipment must be removed without contaminating the healthcare worker or environment

All persons entering the patient room should wear at least:

Respiratory protection with either:

A fit-tested N95 filtering mask and a full face shield or

A powered air-purifying respirator with a full face shield, helmet, or headpiece

Single-use surgical hood that extends to shoulders and fully covers neck

Single-use fluid resistant or impermeable gown and boot covers

Two pairs of single-use nitrile gloves with extended cuffs

Single-use fluid resistant or impermeable apron if patient has vomiting or diarrhea

Institutions may consider enhanced PPE beyond national guidelines

Hospitals should assist and monitor healthcare workers to ensure close adherence to appropriate PPE donning and removal procedures and practices

Once removed, disposable PPE should be discarded in biohazard waste containers, and reusable equipment must be appropriately disinfected before reuse

Hospitals should identify appropriate mechanisms to safely and legally manage and dispose waste suspected to be contaminated with Ebola virus

Healthcare workers with significant exposures

Healthcare workers with skin or mucocutaneous exposure to body fluids from a patient with suspected EVD should immediately wash affected skin surfaces with soap and water, irrigate mucous membranes copiously, and contact appropriate institutional personnel to report a potential exposure

Additional measures

Aerosol-generating procedures, such as noninvasive ventilation, intubation or extubation, and open airway suctioning, should be avoided unless essential and should be conducted in an airborne infection isolation room

Facilities should maintain a log of all persons entering the patient's room

All visitors who have had contact with the patient before hospital admission should be actively monitored for fever and other possible symptoms of EVD

Local public health officials should lead efforts to monitor people exposed to EVD

$\mathrm{EVD}=$ Ebola virus disease, $\mathrm{PPE}=$ personal protective equipment.

to decrease potential transmission (32). The decision whether to transport a potentially infectious patient to a tertiary care facility is controversial, and risks and benefits of either approach must be weighed; if transport can be safely undertaken $(41,42)$, children may benefit from transport to an institution with a dedicated biocontainment unit or greater pediatric expertise. 


\section{Treatment of Ebola Virus Infection}

There is currently no approved or available treatment with proven efficacy against Ebola virus infection in humans. Several promising investigational drugs are in development with limited data from nonhuman primate studies. Postexposure prophylaxis regimens consisting of monoclonal antibodies against virus glycoproteins or small-interfering RNA targeting viral proteins have shown promising results in macaques $(43,44)$, and monoclonal antibody cocktails have demonstrated efficacy treating nonhuman primates after development of symptoms $(45,46)$. Clinicians may decide to offer patients treatment with an Ebola investigational therapeutic on a compassionate use basis as long as appropriate ethical criteria are met and scientifically relevant data are shared to establish the safety and effectiveness of the treatment (47); in those cases, Ebola antiviral medications may be supplied by manufacturers if supply is adequate (48-52) and use must be approved by the appropriate regulatory body $(53,54)$. There is limited experience with the use of convalescent plasma to treat EVD in humans $(55,56)$. The World Health Organization has prioritized investigation of convalescent plasma and whole blood therapy for EVD and issued guidance for appropriate use of these products, recommending transfusion of $10 \mathrm{~mL} / \mathrm{kg}$ whole blood or plasma in children with confirmed EVD with standardized data collection to allow assessment of efficacy $(57,58)$. No Ebola vaccines have been approved for clinical use, although human safety testing of investigational vaccines has been expedited in response to the 2014 West African Ebola virus outbreak (59). Ideally, investigational therapies for patients with EVD, including convalescent plasma, should be used in the context of randomized clinical trials.

\section{Monitoring}

Due to extremely high gastrointestinal and insensible fluid losses, fluid intake and output must be closely monitored. A nasogastric tube may be placed in patients without hemorrhagic manifestations. Serum electrolytes should be measured at least daily to avoid development of life-threatening hyponatremia, hypokalemia, hypocalcemia, hypomagnesemia, and metabolic acidosis, which may occur in patients with severe diarrhea. Patients should be monitored for development of liver failure and for renal failure or cerebral edema in severe cases of EVD. Coagulopathy due to disseminated intravascular coagulation and hepatic dysfunction can occur. Thus, clinicians should assess for coagulopathy and clinical bleeding. Children with severe sepsis or septic shock should be monitored closely for development of cardiac compromise, especially ventricular dysfunction.

Clinicians should consider early placement of a central venous catheter or peripherally inserted central catheter to decrease the risk of exposure to blood from multiple phlebotomies and IV catheter placements. Arterial catheters may present unacceptably high risk of bleeding and healthcare worker exposure; hence, consideration of other forms of monitoring and access for blood sampling is advised.

\section{Supportive Care}

Patients with EVD may present with hypovolemia requiring aggressive initial volume resuscitation. Oral fluid replacement is preferred in patients who are able to drink without vomiting, but administration of IV fluids may be necessary. Hypovolemia from profound vomiting and diarrhea may be exacerbated by capillary leak associated with immune dysregulation, and ongoing significant volume resuscitation to maintain normovolemia may be necessary. Patients may benefit from volume resuscitation with balanced fluids rather than saline (60). Patients who develop severe sepsis or septic shock should be managed according to international sepsis treatment guidelines (61). This includes early goal-directed therapy, vasopressor and inotropic support as needed, glucose control, and enteral nutrition as tolerated. Coagulopathy should be corrected if severe or associated with significant bleeding (61). Given the prominence of immune dysregulation in severe and fatal EVD, clinicians may consider employing sepsis treatment guidelines in any patient with severe EVD.

Although pulmonary involvement of EVD is not common, respiratory failure may occur due to pulmonary edema, circulatory overload, or acute lung injury related to blood transfusion or sepsis. Noninvasive ventilation may not be advisable due to significant droplet dispersion and associated risk to healthcare workers. Furthermore, elective tracheal intubation will likely result in lower risk to the patient and healthcare team than urgent intubation in the setting of aspiration or frank respiratory failure. In addition, airway protection may be required for patients with severe neurologic or bleeding sequelae. Noninvasive ventilation and tracheal intubation are aerosol-generating procedures requiring airborne precautions. Patients should be intubated by an experienced, skilled provider in order to minimize the risk of airway trauma and healthcare provider exposure.

Corticosteroids should generally not be administered to patients with EVD. However, as EVD can cause adrenocortical infection and necrosis (7), clinicians may consider stressdose glucocorticoid administration in patients with persistent shock who require escalating or persistent vasoactive support or replacement mineralocorticoids in patients with inappropriate urine sodium losses.

Due to the risk of secondary bacterial infections and the difficulty of clinical detection in the setting of a severe systemic viral infection, broad-spectrum antibiotics may be administered initially for patients with manifestations of possible sepsis. Antipyresis, analgesia, and antiemetic treatment may provide symptomatic relief; nonsteroidal anti-inflammatory drugs should be avoided due to bleeding risk. Psychosocial support of children and their caregivers are essential components of high-quality care for patients infected with highly virulent emerging pathogens such as Ebola virus; video conferencing and other technology may facilitate such support without increasing risk of virus transmission.

The potential benefits of each treatment must be weighed against both the risks to the patient and healthcare providers. Testing and procedures with unclear benefit should be avoided. Clinicians may decide to limit the use of plasmapheresis, 
extracorporeal life support, cardiopulmonary resuscitation in patients with multiple organ failure, and other high-risk procedures to situations where they show clear evidence of benefit for patients with EVD. In addition, emergent procedures may be inadvisable given the long time required to properly don personal protective equipment, and loss of a controlled environment during emergency procedures will likely create unacceptable risk of transmission to healthcare workers. Institutional bioethics experts may help guide decision making in situations where clinicians are considering resource restriction or nonstandard allocation. As new data become available regarding the efficacy and exposure risk of such procedures in the patient with EVD, recommendations regarding avoiding certain procedures may evolve.

\section{Case Reporting and Documentation}

Little is known about EVD in children in resource-rich settings, and clinicians should strongly consider prospective, systematic detailed case documentation to increase our collective knowledge about EVD (62). The World Health Organization and the International Severe Acute Respiratory and Emerging Infection Consortium have developed a case reporting and biological sampling protocol for emerging severe respiratory pathogens, including informed consent documents and case record forms (63, 64); this protocol can serve as a template for efforts to efficiently and systematically gather information about EVD. Careful case reporting can improve knowledge of not only EVD and its clinical manifestations but also of the effects of treatment strategies.

\section{CONCLUSION}

EVD is a severe multisystem disease with high mortality in children and adults. Hospitals and clinicians must prepare to provide care for patients with EVD before such patients present for care, with particular attention to rigorous infection control in order to limit secondary cases. Although there is no proven specific treatment for EVD, meticulous supportive care offers patients the best chance of survival.

\section{REFERENCES}

1. Baize S, Pannetier D, Oestereich L, et al: Emergence of Zaire Ebola virus disease in Guinea. N Engl J Med 2014; 371:1418-1425

2. McElroy AK, Erickson BR, Flietstra TD, et al: Biomarker correlates of survival in pediatric patients with Ebola virus disease. Emerg Infect Dis $2014 ; 20: 1683-1690$

3. WHO Ebola Response Team: Ebola virus disease in West Africa-the first 9 months of the epidemic and forward projections. N Engl J Med 2014; 371:1481-1495

4. Leroy EM, Kumulungui $B$, Pourrut $X$, et al: Fruit bats as reservoirs of Ebola virus. Nature 2005; 438:575-576

5. Pourrut X, Souris M, Towner JS, et al: Large serological survey showing cocirculation of Ebola and Marburg viruses in Gabonese bat populations, and a high seroprevalence of both viruses in Rousettus aegyptiacus. BMC Infect Dis 2009; 9:159

6. Leroy EM, Rouquet $P$, Formenty $P$, et al: Multiple Ebola virus transmission events and rapid decline of central African wildlife. Science 2004; 303:387-390

7. Feldmann H, Geisbert TW: Ebola haemorrhagic fever. Lancet 2011; 377:849-862
8. Georges AJ, Leroy EM, Renaut AA, et al: Ebola hemorrhagic fever outbreaks in Gabon, 1994-1997: Epidemiologic and health control issues. J Infect Dis 1999; 179(Suppl 1):S65-S75

9. Report of an International Commission: Ebola haemorrhagic fever in Zaire, 1976. Bull World Health Organ 1978; 56:271-293

10. Harcourt BH, Sanchez A, Offermann MK: Ebola virus selectively inhibits responses to interferons, but not to interleukin-1 beta, in endothelial cells. J Virol 1999; 73:3491-3496

11. Sanchez A, Geisbert TW, Feldmann H: Chapter 40. Filoviridae: Marburg and Ebola viruses. In: Fields Virology. Knipe DM, Howley PM (Eds). Fifth Edition. Philadelphia, PA, Wolters Kluwer Health/ Lippincott Williams \& Wilkins, 2007

12. Wong G, Kobinger GP, Qiu X: Characterization of host immune responses in Ebola virus infections. Expert Rev Clin Immunol 2014; 10:781-790

13. Hutchinson KL, Rollin PE: Cytokine and chemokine expression in humans infected with Sudan Ebola virus. J Infect Dis 2007; 196(Suppl 2):S357-S363

14. Baize S, Leroy EM, Georges-Courbot MC, et al: Defective humoral responses and extensive intravascular apoptosis are associated with fatal outcome in Ebola virus-infected patients. Nat Med 1999; 5:423-426

15. Baize S, Leroy EM, Georges AJ, et al: Inflammatory responses in Ebola virus-infected patients. Clin Exp Immunol 2002; 128:163-168

16. Sanchez A, Lukwiya $M$, Bausch $D$, et al: Analysis of human peripheral blood samples from fatal and nonfatal cases of Ebola (Sudan) hemorrhagic fever: Cellular responses, virus load, and nitric oxide levels. J Virol 2004; 78:10370-10377

17. McElroy AK, Erickson BR, Flietstra TD, et al: Ebola hemorrhagic fever: Novel biomarker correlates of clinical outcome. J Infect Dis 2014; 210:558-566

18. Bwaka MA, Bonnet MJ, Calain P, et al: Ebola hemorrhagic fever in Kikwit, Democratic Republic of the Congo: Clinical observations in 103 patients. J Infect Dis 1999; 179(Suppl 1):S1-S7

19. Schulte W, Bernhagen J, Bucala R: Cytokines in sepsis: Potent immunoregulators and potential therapeutic targets-an updated view. Mediators Inflamm 2013; 2013:165974

20. Mupere E, Kaducu OF, Yoti Z: Ebola haemorrhagic fever among hospitalised children and adolescents in northern Uganda: Epidemiologic and clinical observations. Afr Health Sci 2001; 1:60-65

21. Richards GA, Murphy S, Jobson R, et al: Unexpected Ebola virus in a tertiary setting: Clinical and epidemiologic aspects. Crit Care Med 2000; 28:240-244

22. Towner JS, Rollin PE, Bausch DG, et al: Rapid diagnosis of Ebola hemorrhagic fever by reverse transcription-PCR in an outbreak setting and assessment of patient viral load as a predictor of outcome. J Viro/ 2004; 78:4330-4341

23. Report of a WHO/International Study Team: Ebola haemorrhagic fever in Sudan, 1976. Bull World Health Organ 1978; 56:247-270

24. Rowe AK, Bertolli J, Khan AS, et al: Clinical, virologic, and immunologic follow-up of convalescent Ebola hemorrhagic fever patients and their household contacts, Kikwit, Democratic Republic of the Congo. Commission de Lutte contre les Epidémies à Kikwit. J Infect Dis 1999; 179(Suppl 1):S28-S35

25. Bausch DG, Towner JS, Dowell SF, et al: Assessment of the risk of Ebola virus transmission from bodily fluids and fomites. J Infect Dis 2007; 196(Suppl 2):S142-S147

26. Rodriguez LL, De Roo A, Guimard Y, et al: Persistence and genetic stability of Ebola virus during the outbreak in Kikwit, Democratic Republic of the Congo, 1995. J Infect Dis 1999; 179(Suppl 1):S170-S176

27. Centers for Disease Control and Prevention: Ebola virus disease (Ebola): Algorithm for evaluation of the returned traveler. 2014. Available at: http://www.cdc.gov/vhf/ebola/pdf/ebola-algorithm.pdf. Accessed October 14, 2014

28. Centers for Disease Control and Prevention: Epidemiologic risk factors to consider when evaluating a person for exposure to Ebola virus. Available at: http://www.cdc.gov/vhf/ebola/exposure/risk-factors-whenevaluating-person-for-exposure.html. Accessed October 30, 2014

29. Centers for Disease Control and Prevention: Ebola (Ebola virus disease): Information for health care workers and settings. Available at: 
http://www.cdc.gov/vhf/ebola/hcp/index.html. Accessed October 30, 2014

30. Public health Agency of Canada: Ebola virus disease (EVD)-health professionals. Available at: http://www.phac-aspc.gc.ca/id-mi/vhffvh/ebola-professionals-professionnels-eng.php. Accessed October 15,2014

31. Canadian Critical Care Society, Canadian Association of Emergency Physicians, Association of Medical Microbiology \& Infectious Diseases Canada: Ebola clinical care guidelines: A guide for clinicians in Canada. Interim report. Available at: http://www.ammi.ca/ media/69846/Ebola\%20Clinical\%20Care\%20Guidelines\%20 2\%20Sep\%202014.pdf. Accessed October 19, 2014

32. Centers for Disease Control and Prevention: Preparing for Ebola: What U.S. hospitals can learn from Emory Healthcare and Nebraska Medical Center. Available at: http://emergency.cdc.gov/coca/transcripts/2014/ call-transcript-101414.asp. Accessed October 18, 2014

33. Emory Healthcare: Emory Healthcare Ebola preparedness protocols. Available at: http://www.emoryhealthcare.org/ebola-protocol/ehcmessage.html. Accessed October 20, 2014

34. Centers for Disease Control and Prevention: Detailed hospital checklist for Ebola preparedness. Available at: http://www.cdc.gov/ vhf/ebola/pdf/hospital-checklist-ebola-preparedness.pdf. Accessed October 15, 2014

35. Centers for Disease Control and Prevention: Safe management of patients with Ebola virus disease (EVD) in U.S. hospitals. Available at: http://www.cdc.gov/vhf/ebola/hcp/patient-management-us-hospitals.html. Accessed October 15, 2014

36. Centers for Disease Control and Prevention: Guidance on personal protective equipment to be used by healthcare workers during management of patients with Ebola virus disease in U.S. hospitals, including procedures for putting on (donning) and removing (doffing). Available at: http://www.cdc.gov/vhf/ebola/hcp/procedures-for-ppe. Accessed October 23, 2014

37. Centers for Disease Control and Prevention: Infection prevention and control recommendations for hospitalized patients with known or suspected Ebola hemorrhagic fever in U.S. hospitals. Available at: http://www.cdc.gov/vhf/ebola/hcp/infection-prevention-and-controlrecommendations.html. Accessed October 23, 2014

38. World Health Organization: Infection prevention and control guidance for care of patients in health-care settings, with focus on Ebola. Available at: http://apps.who.int/iris/bitstream/10665/130596/1/ WHO_HIS_SDS_2014.4_eng.pdf?ua $=1 \& u a=1 \& u a=1$. Accessed October 15, 2014

39. Centers for Disease Control and Prevention: Interim guidance for specimen collection, transport, testing, and submission for patients with suspected infection with Ebola virus disease. Available at: http://www.cdc. gov/vhf/ebola/hcp/interim-guidance-specimen-collection-submissionpatients-suspected-infection-ebola.html. Accessed October 15, 2014

40. Centers for Disease Control and Prevention: Interim guidance for environmental infection control in hospitals for Ebola virus. Available at: http://www.cdc.gov/vhf/ebola/hcp/environmental-infection-control-in-hospitals.html. Accessed October 15, 2014

41. Centers for Disease Control and Prevention: Interim guidance for emergency medical services (EMS) systems and 9-1-1 public safety answering points (PSAPs) for management of patients with known or suspected Ebola virus disease in the United States. Available at: http://www.cdc. gov/vhf/ebola/hcp/interim-guidance-emergency-medical-services-systems-911-public-safety-answering-points-management-patients-knownsuspected-united-states.html. Accessed October 15, 2014

42. Centers for Disease Control and Prevention: Guidance on air medical transport for patients with Ebola virus. Available at: http://www. cdc.gov/vhf/ebola/hcp/guidance-air-medical-transport-patients.html. Accessed October 15, 2014

43. Olinger GG Jr, Pettitt J, Kim D, et al: Delayed treatment of Ebola virus infection with plant-derived monoclonal antibodies provides protection in rhesus macaques. Proc Natl Acad Sci U S A 2012; 109:18030-18035

44. Geisbert TW, Lee AC, Robbins M, et al: Postexposure protection of non-human primates against a lethal Ebola virus challenge with RNA interference: A proof-of-concept study. Lancet 2010; 375:1896-1905
45. Qiu X, Wong G, Audet J, et al: Reversion of advanced Ebola virus disease in nonhuman primates with ZMapp. Nature 2014; 514:47-53

46. Pettitt J, Zeitlin L, Kim do H, et al: Therapeutic intervention of Ebola virus infection in rhesus macaques with the MB-003 monoclonal antibody cocktail. Sci Trans/ Med 2013; 5:199ra113

47. World Health Organization: Ethical considerations for the use of unregistered interventions for Ebola viral disease; report of an advisory panel to WHO. Available at: http://apps.who.int/iris/bitstream/10665/130997/1/WHO_HIS_KER_GHE_14.1_eng.pdf. Accessed October 15, 2014

48. Mapp biopharmaceutical. Available at: http://www.mappbio.com/. Accessed October 15, 2014

49. Tekmira Pharmaceuticals Corporation: TKM-Ebola. Available at: http://www.tekmira.com/pipeline/tkm-ebola.php. Accessed October 15,2014

50. Sarepta Therapeutics: AVI-7288 for treatment of marburg virus|sarepta therapeutics. Available at: http://www.sarepta.com/ pipeline/avi-7288-marburg. Accessed October 15, 2014

51. BioCryst Pharmaceuticals: BCX4430. Available at: http://www.biocryst.com/bcx_4430. Accessed October 15, 2014

52. Chimerix: Brincidofovir (CMX001). Available at: http://www.chimerix. com/c/discovery-clinical-trials/brincidofovir.php. Accessed October 15,2014

53. Food and Drug Administration: Investigational new drug (IND) application $>$ emergency investigational new drug (EIND) applications for antiviral products. Available at: http://www.fda.gov/Drugs/ DevelopmentApprovalProcess/HowDrugsareDevelopedandApproved/ ApprovalApplications/InvestigationalNewDrugINDApplication/ ucm090039.htm. Accessed October 15, 2014

54. Health Canada: Drugs-special access to drugs and health products. Available at: http://www.hc-sc.gc.ca/dhp-mps/acces/drugs-drogues/ index-eng.php. Accessed October 15, 2014

55. Mupapa K, Massamba M, Kibadi K, et al: Treatment of Ebola hemorrhagic fever with blood transfusions from convalescent patients. International Scientific and Technical Committee. J Infect Dis 1999; 179(Suppl 1):S18-S23

56. Sadek RF, Khan AS, Stevens G, et al: Ebola hemorrhagic fever, Democratic Republic of the Congo, 1995: Determinants of survival. $J$ Infect Dis 1999; 179(Suppl 1):S24-S27

57. World Health Organization: Experimental therapies: Growing interest in the use of whole blood or plasma from recovered Ebola patients (convalescent therapies). Available at: http://www.who.int/mediacentre/news/ebola/26-september-2014/en/. Accessed October 14, 2014

58. World Health Organization: Use of convalescent whole blood or plasma collected from patients recovered from Ebola virus disease. Available at: http://www.who.int/csr/resources/publications/ebola/ convalescent-treatment/en/. Accessed October 14, 2014

59. National Institute of Allergy and Infectious Diseases: NIH to launch human safety study of Ebola vaccine candidate. Available at: http://www.niaid.nih.gov/news/newsreleases/2014/Pages/ EbolaVaxCandidate.aspx. Accessed October 15, 2014

60. Raghunathan K, Shaw A, Nathanson B, et al: Association between the choice of IV crystalloid and in-hospital mortality among critically ill adults with sepsis. Crit Care Med 2014; 42:1585-1591

61. Dellinger RP, Levy MM, Rhodes A, et al; Surviving Sepsis Campaign Guidelines Committee including the Pediatric Subgroup: Surviving sepsis campaign: International guidelines for management of severe sepsis and septic shock: 2012. Crit Care Med 2013; 41:580-637

62. Lurie N, Manolio T, Patterson AP, et al: Research as a part of public health emergency response. N Engl J Med 2013; 368:1251-1255

63. Dunning JW, Merson L, Rohde GG, et al; ISARIC Working Group 3, ISARIC Council: Open source clinical science for emerging infections. Lancet Infect Dis 2014; 14:8-9

64. ISARIC/WHO severe acute respiratory infection biological sampling study. Available at: http://www.prognosis.org/isaric. Accessed October 15, 2014

65. Healthcare Infection Control Practices Advisory Committee: 2007 Guideline for isolation precautions: Preventing transmission of infectious agents in healthcare settings. Available at: http://www.cdc.gov/ hicpac/2007IP/2007ip_part2.html\#e. Accessed October 16, 2014 